\title{
Improving Delay-Range-Dependent Stability Condition for Systems with Interval Time-Varying Delay
}

\author{
Wei Qian, ${ }^{1}$ Tao $\mathrm{Li}^{2}{ }^{2}$ and Juan $\mathrm{Liu}^{3}$ \\ ${ }^{1}$ School of Electrical Engineering and Automation, Henan Polytechnic University, Jiaozuo, Henan 454000, China \\ ${ }^{2}$ School of Automation Engineering, Nanjing University of Aeronautics and Astronautics, Nanjing 210016, China \\ ${ }^{3}$ Department of Mathematics and Information Science, Henan Polytechnic University, Jiaozuo, Henan 454000, China
}

Correspondence should be addressed to Wei Qian; qwei@hpu.edu.cn

Received 26 August 2013; Accepted 12 October 2013

Academic Editor: Bo-Chao Zheng

Copyright (C) 2013 Wei Qian et al. This is an open access article distributed under the Creative Commons Attribution License, which permits unrestricted use, distribution, and reproduction in any medium, provided the original work is properly cited.

\begin{abstract}
This paper discusses the delay-range-dependent stability for systems with interval time-varying delay. Through defining the new Lyapunov-Krasovskii functional and estimating the derivative of the LKF by introducing new vectors, using free matrices and reciprocally convex approach, the new delay-range-dependent stability conditions are obtained. Two well-known examples are given to illustrate the less conservatism of the proposed theoretical results.
\end{abstract}

\section{Introduction}

It is well known that time-varying delays are frequently encountered in many practical control systems, and they are usually regarded as a source of instability and poor performance. So the stability issue of time delay systems has received considerable attention [1-16]. In the last years, in order to further reduce conservatism of the stability results, some methods were developed, such as the delay-fraction approach in $[1,2]$, free weighting matrices method in $[3,4]$, the convex analysis method in $[5,6]$, the reciprocally convex approach in [7], LKF constructing method with matrices that depend on the time delays [8], LKF constructing method with triple-integral terms in [9], LKF constructing method with quadruple-integral terms in [10], and simple LKF having quadratic terms multiplied by a higher degree scalar function $[11,12]$. These methods reduced the conservatism of the stability results. But when delay is interval time-varying, the information of the delay derivative is not full used, which causes the conservatism of the stability results.

Motivated by recent methods, in this paper, we further discuss the stability of linear systems with interval timevarying delay. Firstly, a novel LKF is introduced. Then, by introducing new vectors, using free matrices and reciprocally convex approach, the derivative of LKF is estimated less conservatively, and as a result, the stability criterion is obtained in terms of LMI. Finally, two examples are given to illustrate the effectiveness of the proposed method.

Throughout the note, the used notations are standard. $\mathbf{R}^{n}$ denotes the $n$-dimensional Euclidean space, $\mathbf{R}^{n \times m}$ is a set of $n \times m$ real matrix, $A^{T}$ is the transpose of $A, P>0(P<0)$ means symmetric positive (negative) definite matrix, and $*$ in the matrix denotes the symmetric element; $I$ is the identity matrix of appropriate dimensions, $x_{t}=x(t+\theta), \theta \in[-h, 0]$. Matrices, if their dimensions are not explicitly stated, are assumed to be compatible for algebraic operations.

\section{Problem Formulations}

Consider the following time-delay system:

$$
\begin{gathered}
\dot{x}(t)=A x(t)+A_{1} x(t-h(t)), \\
x(t)=\phi(t), \quad t \in[-h, 0],
\end{gathered}
$$

where $x(t) \in \mathbf{R}^{n}$ is the state vector, the initial condition $\phi(t)$ is a continuously differentiable vector-valued function, $A, A_{1} \in$ $\mathbf{R}^{n \times n}$ are known real constant matrices, and $h(t)$ is the timevarying delay satisfying

$$
h_{1} \leq h(t) \leq h_{2}, \quad \dot{h}(t) \leq \mu \leq \infty,
$$

where $h_{1}, h_{2}$, and $\mu$ are constants. 
To obtain the main results, the following lemmas are needed.

Lemma 1 (see [9]). For any symmetric matrix $Z=Z^{T} \geq 0$, scalars $h_{2}>h_{1}>0$ such that the integration is well defined; then

$$
\begin{aligned}
-\left(h_{2}-h_{1}\right) \int_{-h_{2}}^{-h_{1}} \int_{t+\theta}^{t} x^{T}(s) Z x(s) d s d \theta \\
\quad \leq-\int_{-h_{2}}^{-h_{1}} \int_{t+\theta}^{t} x^{T}(s) d s d \theta Z \int_{-h_{2}}^{-h_{1}} \int_{t+\theta}^{t} x(s) d s d \theta .
\end{aligned}
$$

Lemma 2 (see [7]). Let $f_{1}, f_{2}, \ldots, f_{N}: \mathbf{R}^{m} \rightarrow \mathbf{R}$ have positive values in an open subset $\mathbf{D}$ of $\mathbf{R}^{m}$. Then, the reciprocally convex combination of $f_{i}$ over $\mathbf{D}$ satisfies

$$
\begin{array}{cc}
\min _{\left\{\alpha_{i} \mid \alpha_{i}>0, \sum \alpha_{i}=1\right\}} & \sum_{i} \frac{1}{\alpha_{i}} f_{i}(t)=\sum_{i} f_{i}(t)+\max _{g_{i j}(t)} \sum_{i \neq j} g_{i j}(t) \\
\text { subject to } \quad g_{i, j}: & \mathbf{R}^{m} \longrightarrow \mathbf{R}, \quad g_{i j}(t)=g_{j i}(t), \\
& {\left[\begin{array}{ll}
f_{i}(t) & g_{i j}(t) \\
g_{i j}(t) & f_{j}(t)
\end{array}\right] \geq 0 .}
\end{array}
$$

Lemma 3 (see [16]). The symmetric appropriately dimensional matrices $\Theta>0, \Xi, \Upsilon$, if $\Xi-\Upsilon \Theta \Upsilon^{T}<0$ hold; then there exists a matrix of appropriate dimension $\Pi$ such that the following LMI holds:

$$
\left[\begin{array}{cc}
\Xi+\Upsilon \Pi^{T}+\Pi \Upsilon^{T} & \Pi \\
* & -\Theta
\end{array}\right]<0
$$

\section{Main Results}

In this section, the stability of system (1) is investigated. Through constructing a novel LKF and estimating the derivative of it, new stability condition is provided.
Firstly, define the following vector as

$$
\begin{aligned}
& \zeta^{T}(t)=\left[x^{T}(t) \int_{t-h_{1}}^{t} x^{T}(s) d s\right],
\end{aligned}
$$

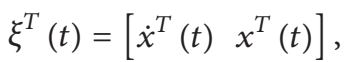

and then construct the L-K functional as follows:

$$
V\left(x_{t}\right)=\sum_{i=1}^{4} V_{i}\left(x_{t}\right),
$$

where

$$
\begin{aligned}
& V_{1}\left(x_{t}\right)=\zeta^{T}(t) P \zeta(t), \\
& V_{2}\left(x_{t}\right)= \int_{t-h_{1}}^{t} x^{T}(s) Q_{1} x(s) d s+\int_{t-h(t)}^{t-h_{1}} x^{T}(s) Q_{2} x(s) d s \\
&+\int_{t-h_{2}}^{t-h(t)} x^{T}(s) Q_{3} x(s) d s, \\
& V_{3}\left(x_{t}\right)= h_{1} \int_{-h_{1}}^{0} \int_{t+\theta}^{t} \xi^{T}(s)\left[\begin{array}{cc}
R_{1} & 0 \\
* & R_{2}
\end{array}\right] \xi(s) d s d \theta \\
&+\left(h_{2}-h_{1}\right) \int_{-h_{2}}^{-h_{1}} \int_{t+\theta}^{t} \xi^{T}(s)\left[\begin{array}{cc}
X_{1} & 0 \\
* & X_{2}
\end{array}\right] \xi(s) d s d \theta, \\
& V_{4}\left(x_{t}\right)= \frac{h_{1}^{2}}{2} \int_{-h_{1}}^{0} \int_{\theta}^{0} \int_{t+\lambda}^{t} \dot{x}^{T}(s) U_{1} \dot{x}(s) d s d \lambda d \theta \\
&+\frac{h_{2}^{2}-h_{1}^{2}}{2} \int_{-h_{1}}^{-h_{2}} \int_{\theta}^{0} \int_{t+\lambda}^{t} \dot{x}^{T}(s) U_{2} \dot{x}(s) d s d \lambda d \theta \\
&+\frac{1}{2} \int_{t-h_{2}}^{t-h_{1}} \int_{\theta}^{t-h_{1}} \int_{\lambda}^{t} \dot{x}^{T}(s) U_{3} \dot{x}(s) d s d \lambda d \theta,
\end{aligned}
$$

and matrices $Q_{1}, Q_{2}, Q_{3}, R_{1}, R_{2}, X_{1}, X_{2}, U_{1}, U_{2}, U_{3} \in \mathbf{R}^{n \times n}>$ $0, P=\left[P_{i j}\right](1 \leq i \leq j \leq 2) \in \mathbf{R}^{2 n \times 2 n}>0$.

In order to make the proof process clear, the following notations are used:

$$
\begin{aligned}
& \alpha(t)=h(t)-h_{1}, \quad \beta(t)=h_{2}-h(t), \quad \varphi(t)=\frac{1}{\alpha(t)} \int_{t-h(t)}^{t-h_{1}} x^{T}(s) d s, \quad \phi(t)=\frac{1}{\beta(t)} \int_{t-h_{2}}^{t-h(t)} x^{T}(s) d s, \\
& \chi^{T}(t)=\left[\begin{array}{lllllllll}
x^{T}(t) & x^{T}(t-h(t)) & x^{T}\left(t-h_{1}\right) & x^{T}\left(t-h_{2}\right) & \int_{t-h_{1}} x^{T}(s) & \varphi^{T}(t) & \phi^{T}(t) & \dot{x}^{T}(t)
\end{array}\right]
\end{aligned}
$$

and block entry matrices $e_{i}(i=1,2, \ldots, 5)$ (e.g., $e_{2}^{T}=$ $\left[\begin{array}{llllllll}0 & I & 0 & 0 & 0 & 0 & 0 & 0\end{array}\right]$ ).

Then taking the time derivatives of $V_{i}\left(x_{t}\right)$ along the trajectory of system (1) yields

$$
\begin{gathered}
\dot{V}_{1}\left(x_{t}\right)=2 \zeta^{T}(t) P \dot{\zeta}(t)=2 \chi^{T}(t)\left[\begin{array}{ll}
e_{1} & e_{5}
\end{array}\right] P\left[\begin{array}{ll}
e_{8} & e_{1}-e_{3}
\end{array}\right]^{T} \chi(t), \\
\dot{V}_{2}\left(x_{t}\right)=x^{T}(t) Q_{1} x(t)-x^{T}\left(t-h_{1}\right)\left(Q_{1}-Q_{2}\right) x\left(t-h_{1}\right)
\end{gathered}
$$

$$
\begin{gathered}
-(1-\dot{h}(t)) x^{T}(t-h(t))\left(Q_{2}-Q_{3}\right) x(t-h(t)) \\
-x^{T}\left(t-h_{2}\right) Q_{3} x\left(t-h_{2}\right) \\
\leq \chi^{T}(t)\left[e_{1} Q_{1} e_{1}^{T}-e_{3}\left(Q_{1}-Q_{2}\right) e_{3}^{T}\right. \\
\left.-(1-\mu) e_{2}\left(Q_{2}-Q_{3}\right) e_{2}^{T}-e_{4} Q_{3} e_{4}^{T}\right] \chi(t),
\end{gathered}
$$

where $Q_{2}-Q_{3}>0$. 
To reduce the conservatism of the main result, by using the Jesen inequality and Lemma 2 with introducing appropriate matrices $Y_{1}, Y_{2} \in \mathbf{R}^{n \times n}$, we can calculate $\dot{V}_{3}\left(x_{t}\right)$ as

$$
\begin{aligned}
\dot{V}_{3}\left(x_{t}\right)= & h_{1}^{2} \xi^{T}(t)\left[\begin{array}{cc}
R_{1} & 0 \\
* & R_{2}
\end{array}\right] \xi(t) \\
& +\left(h_{2}-h_{1}\right)^{2} \xi^{T}(t)\left[\begin{array}{cc}
X_{1} & 0 \\
* & X_{2}
\end{array}\right] \xi(t) \\
& -h_{1} \int_{t-h_{1}}^{t} \xi^{T}(s)\left[\begin{array}{cc}
R_{1} & 0 \\
* & R_{2}
\end{array}\right] \xi(s) d s \\
& -\left(h_{2}-h_{1}\right) \int_{t-h_{2}}^{t-h_{1}} \xi^{T}(s)\left[\begin{array}{cc}
X_{1} & 0 \\
* & X_{2}
\end{array}\right] \xi(s) d s \\
\leq & \chi^{T}(t)\left\{e_{8}\left[h_{1}^{2} R_{1}+\left(h_{2}-h_{1}\right)^{2} X_{1}\right] e_{8}^{T}\right. \\
& \quad+e_{1}\left[h_{1}^{2} R_{2}+\left(h_{2}-h_{1}\right)^{2} X_{2}\right] e_{1}^{T} \\
& \left.\quad-\left(e_{1}-e_{3}\right) R_{1}\left(e_{1}-e_{3}\right)^{T}-e_{5} R_{2} e_{5}^{T}\right\} \chi(t) \\
& -\chi^{T}(t)\left[e_{2}-e_{3} e_{4}-e_{2}\right] \\
& \times\left[\begin{array}{cc}
X_{1} & Y_{1} \\
* & X_{1}
\end{array}\right]\left[e_{2}-e_{3} e_{4}-e_{2}\right]^{T} \chi(t)-\Gamma_{1},
\end{aligned}
$$

where by using Lemma 2 with matrix $Y_{1}$, one can get

$$
\begin{aligned}
& -\left(h_{2}-h_{1}\right) \int_{t-h_{2}}^{t-h_{1}} \dot{x}^{T}(s) X_{1} \dot{x}(s) d s \\
& =-\left(h_{2}-h_{1}\right) \int_{t-h(t)}^{t-h_{1}} \dot{x}^{T}(s) X_{1} \dot{x}(s) d s \\
& -\left(h_{2}-h_{1}\right) \int_{t-h_{2}}^{t-h(t)} \dot{x}^{T}(s) X_{1} \dot{x}(s) d s \\
& \leq-\left[\begin{array}{l}
x(t-h(t))-x\left(t-h_{1}\right) \\
x\left(t-h_{2}\right)-x(t-h(t))
\end{array}\right]^{T} \\
& \times\left[\begin{array}{cc}
X_{1} & Y_{1} \\
* & X_{1}
\end{array}\right]\left[\begin{array}{l}
x(t-h(t))-x\left(t-h_{1}\right) \\
x\left(t-h_{2}\right)-x(t-h(t))
\end{array}\right] \\
& =-\chi^{T}(t)\left[e_{2}-e_{3} e_{4}-e_{2}\right]\left[\begin{array}{cc}
X_{1} & Y_{1} \\
* & X_{1}
\end{array}\right] \\
& \times\left[\begin{array}{ll}
e_{2}-e_{3} & e_{4}-e_{2}
\end{array}\right]^{T} \chi(t),
\end{aligned}
$$

and by using Lemma 2 with matrix $Y_{2}$, one can get

$$
\begin{aligned}
-\left(h_{2}-h_{1}\right) \int_{t-h_{2}}^{t-h_{1}} x^{T}(s) X_{2} x(s) d s \\
=-\left(h_{2}-h_{1}\right) \int_{t-h(t)}^{t-h_{1}} x^{T}(s) X_{2} x(s) d s \\
-\left(h_{2}-h_{1}\right) \int_{t-h_{2}}^{t-h(t)} x^{T}(s) X_{2} x(s) d s
\end{aligned}
$$

$$
\begin{aligned}
\leq & -\left[\begin{array}{l}
\int_{t-h(t)}^{t-h_{1}} x(s) d s \\
\int_{t-h_{2}}^{t-h(t)} x(s) d s
\end{array}\right]^{T} \\
& \times\left[\begin{array}{cc}
X_{2} & Y_{2} \\
* & X_{2}
\end{array}\right]\left[\begin{array}{l}
\int_{t-h(t)}^{t-h_{1}} x(s) d s \\
\int_{t-h_{2}}^{t-h(t)} x(s) d s
\end{array}\right]=\Gamma_{1} .
\end{aligned}
$$

To the time-derivative of $V_{4}\left(x_{t}\right)$, it can be calculated as

$$
\begin{aligned}
\dot{V}_{4}\left(x_{t}\right)= & \frac{h_{1}^{4}}{4} \dot{x}^{T}(t) U_{1} \dot{x}(t)+\frac{\left(h_{2}^{2}-h_{1}^{2}\right)^{2}}{4} \dot{x}^{T}(t) U_{2} \dot{x}(t) \\
& +\frac{\left(h_{2}-h_{1}\right)^{2}}{4} \dot{x}^{T}(t) U_{3} \dot{x}(t) \\
& -\frac{h_{1}^{2}}{2} \int_{-h_{1}}^{0} \int_{t+\theta}^{t} \dot{x}^{T}(s) U_{1} \dot{x}(s) d s d \theta \\
& -\frac{h_{2}^{2}-h_{1}^{2}}{2} \int_{-h_{2}}^{-h_{1}} \int_{t+\theta}^{t} \dot{x}^{T}(s) U_{2} \dot{x}(s) d s d \theta \\
& -\frac{1}{2} \int_{t-h_{2}}^{t-h_{1}} \int_{\theta}^{t-h_{1}} \dot{x}^{T}(s) U_{3} \dot{x}(s) d s d \theta \\
= & \chi^{T}(t) e_{8}\left[\frac{h_{1}^{2}}{2} U_{1}+\frac{\left(h_{2}^{2}-h_{1}^{2}\right)^{2}}{4} U_{2}\right. \\
& \left.\quad+\frac{\left(h_{2}-h_{1}\right)^{2}}{4} U_{3}\right] e_{8}^{T} \chi(t) \\
& -\chi^{T}(t)\left(h_{1} e_{1}-e_{5}\right) U_{1}\left(h_{1} e_{1}-e_{5}\right)^{T} \chi(t) \\
& -\chi^{T}(t)\left[\left(e_{3}-e_{6}\right)^{T} U_{3}\left(e_{3}-e_{6}\right)\right. \\
& \left.\quad-\left(e_{2}-e_{7}\right)^{T} U_{3}\left(e_{2}-e_{7}\right)\right] \chi(t)-\Gamma_{2},
\end{aligned}
$$

where by using Lemma 1, we can get

$$
\begin{gathered}
-\frac{h_{1}^{2}}{2} \int_{-h_{1}}^{0} \int_{t+\theta}^{t} \dot{x}^{T}(s) U_{1} \dot{x}(s) d s d \theta \\
\leq-\left[h_{1} x(t)-\int_{t-h_{1}}^{t} x(s) d s\right]^{T} \\
\quad \times U_{1}\left[h_{1} x(t)-\int_{t-h_{1}}^{t} x(s) d s\right] \\
=-\chi^{T}(t)\left(h_{1} e_{1}-e_{5}\right) U_{1}\left(h_{1} e_{1}-e_{5}\right)^{T} \chi(t), \\
-\frac{1}{2} \int_{t-h_{2}}^{t-h_{1}} \int_{\theta}^{t-h_{1}} \dot{x}^{T}(s) U_{3} \dot{x}(s) d s d \theta
\end{gathered}
$$




$$
\begin{aligned}
& \leq-\frac{1}{2} \int_{t-h(t)}^{t-h_{1}} \int_{\theta}^{t-h_{1}} \dot{x}^{T}(s) U_{3} \dot{x}(s) d s d \theta \\
&-\frac{1}{2} \int_{t-h_{2}}^{t-h(t)} \int_{\theta}^{t-h(t)} \dot{x}^{T}(s) U_{3} \dot{x}(s) d s d \theta \\
& \leq-\left[\left(h(t)-h_{1}\right) x\left(t-h_{1}\right)-\int_{t-h(t)}^{t-h_{1}} x(s) d s\right]^{T} \\
& \times \frac{U_{3}}{\alpha^{2}(t)}\left[\left(h(t)-h_{1}\right) x\left(t-h_{1}\right)-\int_{t-h(t)}^{t-h_{1}} x(s) d s\right] \\
&-\left[\left(h_{2}-h(t)\right) x(t-h(t))-\int_{t-h_{2}}^{t-h(t)} x(s) d s\right]^{T} \\
& \times \frac{U_{3}}{\beta^{2}(t)}\left[\left(h_{2}-h(t)\right) x(t-h(t))-\int_{t-h_{2}}^{t-h(t)} x(s) d s\right] \\
&=-\chi^{T}(t)\left[\left(e_{3}-e_{6}\right)^{T} U_{3}\left(e_{3}-e_{6}\right)\right. \\
&\left.\quad-\left(e_{2}-e_{7}\right)^{T} U_{3}\left(e_{2}-e_{7}\right)\right] \chi(t) .
\end{aligned}
$$

Similar to the method in (12)-(13), by introducing matrix $Y_{3} \in \mathbf{R}^{n \times n}$ and using Lemma 2, we can estimate the following:

$$
\begin{aligned}
-\frac{h_{2}^{2}-h_{1}^{2}}{2} \int_{-h_{2}}^{-h_{1}} \int_{t+\theta}^{t} \dot{x}^{T}(s) U_{2} \dot{x}(s) d s d \theta & -\frac{h_{2}^{2}-h_{1}^{2}}{2} \int_{-h(t)}^{-h_{1}} \int_{t+\theta}^{t} \dot{x}^{T}(s) U_{2} \dot{x}(s) d s d \theta \\
& -\frac{h_{2}^{2}-h_{1}^{2}}{2} \int_{-h_{2}}^{-h(t)} \int_{t+\theta}^{t} \dot{x}^{T}(s) U_{2} \dot{x}(s) d s d \theta \\
\leq & -\left[\begin{array}{c}
\left(h(t)-h_{1}\right) x(t)-\int_{t-h(t)}^{t-h_{1}} x(s) d s \\
\left(h_{2}-h(t)\right) x(t)-\int_{t-h_{2}}^{t-h(t)} x(s) d s
\end{array}\right] \\
& \times\left[\begin{array}{cc}
U_{2} & Y_{3} \\
* & U_{2}
\end{array}\right]\left[\begin{array}{l}
\left(h(t)-h_{1}\right) x(t)-\int_{t-h(t)}^{t-h_{1}} x(s) d s \\
\left(h_{2}-h(t)\right) x(t)-\int_{t-h_{2}}^{t-h(t)} x(s) d s
\end{array}\right]=\Gamma_{2} .
\end{aligned}
$$

In the last, in order to obtain the stability result base on LMI, we can deal with $\Gamma_{1}, \Gamma_{2}$ as follows:

$$
\begin{aligned}
\Gamma_{1}+\Gamma_{2}= & -\chi^{T}(t)\left[\begin{array}{lllllccc}
0 & 0 & 0 & 0 & 0 & \alpha(t) & 0 & 0 \\
0 & 0 & 0 & 0 & 0 & 0 & \beta(t) & 0
\end{array}\right]^{T} \\
& {\left[\begin{array}{cc}
X_{2} & Y_{2} \\
* & X_{2}
\end{array}\right]\left[\begin{array}{lllllccc}
0 & 0 & 0 & 0 & 0 & \alpha(t) & 0 & 0 \\
0 & 0 & 0 & 0 & 0 & 0 & \beta(t) & 0
\end{array}\right] \chi(t) }
\end{aligned}
$$

$$
\begin{aligned}
& -\chi^{T}(t)\left[\begin{array}{cccccccc}
\alpha(t) & 0 & 0 & 0 & 0 & -\alpha(t) & 0 & 0 \\
\beta(t) & 0 & 0 & 0 & 0 & 0 & -\beta(t) & 0
\end{array}\right]^{T} \\
& \times\left[\begin{array}{cc}
U_{2} & Y_{3} \\
* & U_{2}
\end{array}\right] \\
& \times\left[\begin{array}{cccccccc}
\alpha(t) & 0 & 0 & 0 & 0 & -\alpha(t) & 0 & 0 \\
\beta(t) & 0 & 0 & 0 & 0 & 0 & -\beta(t) & 0
\end{array}\right] \chi(t) \\
= & -\chi^{T}(t) \Upsilon(t) \Theta \Upsilon^{T}(t) \chi(t),
\end{aligned}
$$

where

$$
\begin{gathered}
\Upsilon^{T}(t)=\left[\begin{array}{cccccccc}
\alpha(t) & 0 & 0 & 0 & 0 & -\alpha(t) & 0 & 0 \\
\beta(t) & 0 & 0 & 0 & 0 & 0 & -\beta(t) & 0 \\
0 & 0 & 0 & 0 & 0 & \alpha(t) & 0 & 0 \\
0 & 0 & 0 & 0 & 0 & 0 & \beta(t) & 0
\end{array}\right], \\
\Theta=\left[\begin{array}{ccccc}
U_{2} & Y_{3} & 0 & 0 \\
* & U_{2} & 0 & 0 \\
0 & 0 & X_{2} & Y_{2} \\
0 & 0 & * & X_{2}
\end{array}\right] .
\end{gathered}
$$

Also, for appropriate matrices $N_{1}, N_{2}$, one can have

$$
\begin{aligned}
2\left[x^{T}(t) N_{1}+\dot{x}^{T}(t) N_{2}\right] \\
\quad \times\left[A x(t)+A_{1} x(t-h(t))-\dot{x}(t)\right]=0 .
\end{aligned}
$$

Therefore, combining (10)-(19), we can obtain

$$
\dot{V}\left(x_{t}\right)=\sum_{i=1}^{4} \dot{V}_{i}\left(x_{t}\right) \leq \chi^{T}(t)\left[\Xi-\Upsilon(t) \Theta \Upsilon^{T}(t)\right] \chi(t),
$$

where

$$
\begin{aligned}
& \Xi=\left[\begin{array}{ll}
e_{1} & e_{5}
\end{array}\right] P\left[\begin{array}{ll}
e_{8} & e_{1}-e_{3}
\end{array}\right]^{T}+\left[\begin{array}{ll}
e_{8} & e_{1}-e_{3}
\end{array}\right] P\left[\begin{array}{ll}
e_{1} & e_{5}
\end{array}\right]^{T} \\
& -e_{3}\left(Q_{1}-Q_{2}\right) e_{3}^{T}-(1-\mu) e_{2}\left(Q_{2}-Q_{3}\right) e_{2}^{T}-e_{4} Q_{3} e_{4}^{T} \\
& +e_{8}\left[h_{1}^{2} R_{1}+\left(h_{2}-h_{1}\right)^{2} X_{1}+\frac{h_{1}^{2}}{2} U_{1}\right. \\
& \left.+\frac{\left(h_{2}^{2}-h_{1}^{2}\right)^{2}}{4} U_{2}+\frac{\left(h_{2}-h_{1}\right)^{2}}{4} U_{3}\right] e_{8}^{T} \\
& +e_{1}\left[Q_{1}+h_{1}^{2} R_{2}+\left(h_{2}-h_{1}\right)^{2} X_{2}\right] e_{1}^{T} \\
& -\left(e_{1}-e_{3}\right) R_{1}\left(e_{1}-e_{3}\right)^{T}-e_{5} R_{2} e_{5}^{T} \\
& -\left[\begin{array}{ll}
e_{2}-e_{3} & e_{4}-e_{2}
\end{array}\right]\left[\begin{array}{cc}
X_{1} & Y_{1} \\
* & X_{1}
\end{array}\right]\left[\begin{array}{ll}
e_{2}-e_{3} & e_{4}-e_{2}
\end{array}\right]^{T} \\
& -\left(h_{1} e_{1}-e_{5}\right) U_{1}\left(h_{1} e_{1}-e_{5}\right)^{T} \\
& -\left(e_{3}-e_{6}\right)^{T} U_{3}\left(e_{3}-e_{6}\right)-\left(e_{2}-e_{7}\right)^{T} U_{3}\left(e_{2}-e_{7}\right) \\
& +\left(e_{1} N_{1}+e_{8} N_{2}\right)\left(e_{1}^{T} A+e_{2}^{T} A_{1}-e_{8}\right)^{T} \\
& +\left(e_{1}^{T} A+e_{2}^{T} A_{1}-e_{8}\right)\left(e_{1} N_{1}+e_{8} N_{2}\right)^{T} .
\end{aligned}
$$


As we know, if $\Xi-\Upsilon(t) \Theta \Upsilon^{T}(t)<0$ holds, then $\dot{V}\left(x_{t}\right)<0$, which means that system (1) is asymptotically stable. So, by Lemma 3, there exists a matrix of appropriate dimension $\Pi$ such that the following LMI holds:

$$
\left[\begin{array}{cc}
\Xi+\Upsilon \Pi^{T}+\Pi \Upsilon^{T} & \Pi \\
* & -\Theta
\end{array}\right]<0
$$

So, we give the main theorem of this paper as follows.

Theorem 4. The time-delayed system (1) having contrains (2) is asymptotically stable, if there exist matrices $Q_{1}, Q_{2}, Q_{3}, R_{1}, R_{2}, X_{1}, X_{2}, U_{1}, U_{2}, U_{3} \in \mathbf{R}^{n \times n}>0$, matrix $P \in \mathbf{R}^{2 n \times 2 n}>0$, and appropriate matrices $Y_{1}, Y_{2}, Y_{3}, N_{1}, N_{2}, \Pi$ such that the following LMIs hold:

$$
\begin{gathered}
Q_{2}-Q_{3}>0, \\
{\left[\begin{array}{cc}
\Xi+\Upsilon_{1} \Pi^{T}+\Pi \Upsilon_{1}^{T} & \Pi \\
* & -\Theta
\end{array}\right]<0,} \\
{\left[\begin{array}{cc}
\Xi+\Upsilon_{2} \Pi^{T}+\Pi \Upsilon_{2}^{T} & \Pi \\
* & -\Theta
\end{array}\right]<0,}
\end{gathered}
$$

where $\Xi, \Upsilon, \Pi$, and $\Theta$ are defined in (18) and (21).

Proof. From above, one can see that $\Xi+\Upsilon \Pi^{T}+\Pi \Upsilon^{T}$ is a convex combination of matrix $\Pi$ as

$$
\begin{aligned}
\Xi+\Upsilon \Pi^{T}+\Pi \Upsilon^{T}= & \Xi+\left(h(t)-h_{1}\right)\left(\Upsilon_{1} \Pi^{T}+\Pi \Upsilon_{1}^{T}\right) \\
& +\left(h_{2}-h(t)\right)\left(\Upsilon_{2} \Pi^{T}+\Pi \Upsilon_{2}^{T}\right)<0 .
\end{aligned}
$$

So (22) can be handled nonconservatively by the two corresponding boundary LMIs:

$$
\begin{gathered}
{\left[\begin{array}{cc}
\Xi+\Upsilon_{1} \Pi^{T}+\Pi \Upsilon_{1}^{T} & \Pi \\
* & -\Theta
\end{array}\right]<0,} \\
{\left[\begin{array}{cc}
\Xi+\Upsilon_{2} \Pi^{T}+\Pi \Upsilon_{2}^{T} & \Pi \\
* & -\Theta
\end{array}\right]<0,}
\end{gathered}
$$

where $\Upsilon_{1}=\Upsilon\left(h(t)-h_{1}=0\right), \Upsilon_{2}=\Upsilon\left(h_{2}-h(t)=0\right)$. This completes the proof.

Remark 5. From (17) and (22), it can be seen that, in order to obtain the less conservative stability condition in terms of LMI, $\alpha(t), \beta(t)$ are used, but free matrices $\Pi=\left[\Pi_{i j}\right](1 \leq i \leq$ $j \leq 8$ ) are also introduced, which increases the computational burden; this is a disadvantage of the proposed method.

Remark 6. When $h_{1} \leq h(t) \leq h_{2}, \mu_{1} \leq \dot{h}(t) \leq \mu_{2}$, let $\Xi_{1}=\Xi\left(\mu=\mu_{1}\right), \Xi_{2}=\Xi\left(\mu=\mu_{2}\right)$, and using the convex combination again with $\left[\begin{array}{cc}\Xi_{1}+\Upsilon_{1} \Pi^{T}+\Pi \Upsilon_{1}^{T} & \Pi \\ * & -\Theta\end{array}\right]<0,\left[\begin{array}{cc}\Xi_{1}+\Upsilon_{2} \Pi^{T}+\Pi \Upsilon_{2}^{T} & \Pi \\ * & -\Theta\end{array}\right]<$ $0,\left[\begin{array}{cc}\Xi_{2}+\Upsilon_{1} \Pi^{T}+\Pi \Upsilon_{1}^{T} & \Pi \\ * & -\Theta\end{array}\right]<0,\left[\begin{array}{cc}\Xi_{2}+\Upsilon_{2} \Pi^{T}+\Pi \Upsilon_{2}^{T} & \Pi \\ * & -\Theta\end{array}\right]^{*}<0$, the corresponding stability criterion can also be obtained.

\section{Numerical Examples}

In this section, the effectiveness of the obtained results in this paper is shown by the two well-known numerical examples.
TABLE 1: Admissible upper bound $h_{2}$ for various $h_{1}$ and $\mu$ in Example 1.

\begin{tabular}{lcccc}
\hline$h_{1}$ & Methods & $\mu=0.3$ & $\mu=0.5$ & $\mu=0.9$ \\
\hline \multirow{3}{*}{2} & Shao [6] & 2.69 & 2.50 & 2.50 \\
& Sun et al. [9] & 2.91 & 2.50 & 2.50 \\
& Theorem 4 & 3.00 & 2.64 & 2.64 \\
& Shao [6] & 3.25 & 3.25 & 3.25 \\
3 & Sun et al. [9] & 3.34 & 3.34 & 3.34 \\
& Theorem 4 & 3.35 & 3.35 & 3.35 \\
\hline
\end{tabular}

TABLE 2: Admissible upper bound $h_{2}$ for various $h_{1}$ and $\mu=0.3$ in Example 1.

\begin{tabular}{llcccc}
\hline Methods & $h_{1}$ & 0.3 & 0.5 & 0.8 & 1 \\
\hline Shao [6] & $h_{2}$ & 2.22 & 2.22 & 2.23 & 2.24 \\
Sun et al. [9] & $h_{2}$ & 2.26 & 2.28 & 2.30 & 2.30 \\
Theorem 4 & $h_{2}$ & 2.39 & 2.41 & 2.42 & 2.43 \\
\hline
\end{tabular}

Example 1. Consider the linear system (1) with

$$
A=\left[\begin{array}{cc}
-2 & 0 \\
0 & -0.9
\end{array}\right], \quad A_{1}=\left[\begin{array}{cc}
-1 & 0 \\
-1 & -1
\end{array}\right] .
$$

The purpose is to compare the admissible upper bounds $h_{2}$ given lower bound $h_{1}$ and $\mu$.

For $h_{1}=0, \mu=0.5,0.9$, in [14], the result is $h_{2}=$ $2.04,1.37$, in [17], the result is $h_{2}=2.33,1.87$, and our results are $h_{2}=2.33,1.87$.

For $h_{1}=1, \mu=0.5,0.9$, in [14], the result is $h_{2}=$ $2.07,1.74$, in [17], the result is $h_{2}=2.33,2.07$, and our results are $h_{2}=2.43,2.08$.

For $h_{1}=2, \mu=0.5,0.9$, in [14], the result is $h_{2}=$ $2.43,2.43$, in [17], the result is $h_{2}=2.61,2.61$, and our results are $h_{2}=2.64,2.64$.

For various $h_{1}, \mu$, the maximum upper bounds on delay $h_{2}$ by different methods are also listed in Table 1. It can be seen that the result obtained in this paper is less conservative.

Example 2. Consider the linear system (1) with

$$
A=\left[\begin{array}{cc}
0 & 1 \\
-1 & -2
\end{array}\right], \quad A_{1}=\left[\begin{array}{cc}
0 & 0 \\
-1 & 1
\end{array}\right]
$$

The purpose is to compare the admissible upper bounds $h_{2}$ which guarantee the asymptotic stability of the above system for given lower bound $h_{1}$ and $\mu$. This example is used in many recent papers, such as $[6,9]$, For $\mu=0.3$, Table 2 lists the comparison of our results with some recent ones, and it is easy to see that the results obtained in this paper are less conservative.

\section{Conclusions}

In this note, the stability of interval time-varying delay systems has been discussed. Through constructing a novel LKF and using some new analysis methods, the delay-rangedependent stability criteria were derived. Compared with 
some previous stability conditions, the obtained main results in this paper have less conservatism. In the end, numerical examples were given to show the superiority of the obtained criteria and their improvements over the existing results.

\section{Conflict of Interests}

The authors declare that there is no conflict of interests regarding the publication of this paper.

\section{Acknowledgments}

This work is supported by the National Nature Science Foundation under Grants 61104119, the Science and Technology Innovation Talents Project of Henan University under Grant 13HASTIT044, the Doctoral Foundation from Henan Polytechnic University under Grants B2010-50, and the Young Core Instructor Foundation from the Department of Education of Henan Province under Grant 2011GGJS-054.

\section{References}

[1] X. L. Zhu, Y. Wang, and G. H. Yang, "New stability criteria for continuous-time systems with interval time-varying delay," IET Control Theory \& Applications, vol. 4, no. 6, pp. 1101-1107, 2010.

[2] X. Jiang and Q. L. Han, "New stability criteria for linear systems with interval time-varying delay," Automatica, vol. 44, no. 10, pp. 2680-2685, 2008.

[3] Y. He, Q. G. Wang, C. Lin, and M. Wu, "Delay-range-dependent stability for systems with time-varying delay," Automatica, vol. 43, no. 2, pp. 371-376, 2007.

[4] M. J. Park, O. M. Kwon, J. H. Park, and S. M. Lee, "A new augmented Lyapunov-Krasovskii functional approach for stability of linear systems with time-varying delays," Applied Mathematics and Computation, vol. 217, no. 17, pp. 7197-7209, 2011.

[5] P. Park and J. W. Ko, "Stability and robust stability for systems with a time-varying delay," Automatica, vol. 43, no. 10, pp. 18551858, 2007.

[6] H. Shao, "New delay-dependent stability criteria for systems with interval delay," Automatica, vol. 45, no. 3, pp. 744-749, 2009.

[7] P. Park, J. W. Ko, and C. Jeong, "Reciprocally convex approach to stability of systems with time-varying delays," Automatica, vol. 47, no. 1, pp. 235-238, 2011.

[8] E. Fridman, U. Shaked, and K. Liu, "New conditions for delayderivative-dependent stability," Automatica, vol. 45, no. 11, pp. 2723-2727, 2009.

[9] J. Sun, G. P. Liu, J. Chen, and D. Rees, "Improved delay-rangedependent stability criteria for linear systems with time-varying delays," Automatica, vol. 46, no. 2, pp. 466-470, 2010.

[10] W. Qian, S. Cong, T. Li, and S. Fei, "Improved stability conditions for systems with interval time-varying delay," International Journal of Control, Automation, and Systems, vol. 10, no. 6, pp. 1146-1152, 2012.

[11] J. H. Kim, "Note on stability of linear systems with time-varying delay," Automatica, vol. 47, no. 9, pp. 2118-2121, 2011.

[12] M. N. A. Parlakçı, "Extensively augmented Lyapunov functional approach for the stability of neutral time-delay systems," IET Control Theory \& Applications, vol. 2, no. 5, pp. 431-436, 2008.
[13] X. Nian, H. Pan, W. Gui, and H. Wang, "New stability analysis for linear neutral system via state matrix decomposition," Applied Mathematics and Computation, vol. 215, no. 5, pp. 18301837, 2009.

[14] T. Li, L. Guo, and L. Wu, "Simplified approach to the asymptotical stability of linear systems with interval time-varying delay," IET Control Theory \& Applications, vol. 3, no. 2, pp. 252-260, 2009.

[15] Y. Chen, W. X. Zheng, and A. Xue, "A new result on stability analysis for stochastic neutral systems," Automatica, vol. 46, no. 12, pp. 2100-2104, 2010.

[16] J. Liu and J. Zhang, "Note on stability of discrete-time timevarying delay systems," IET Control Theory \& Applications, vol. 6, no. 2, pp. 335-339, 2012.

[17] W. Zhang, X. Cai, and Z. Z. Han, "Robust stability criteria for systems with interval time-varying delay and nonlinear perturbations," Journal of Computational and Applied Mathematics, vol. 234, no. 1, pp. 174-180, 2010. 


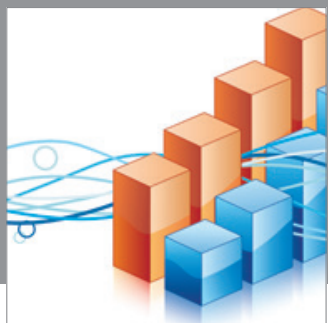

Advances in

Operations Research

mansans

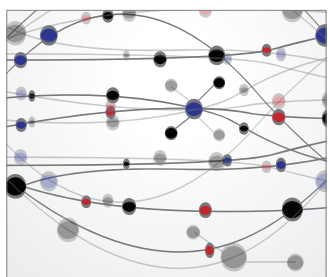

The Scientific World Journal
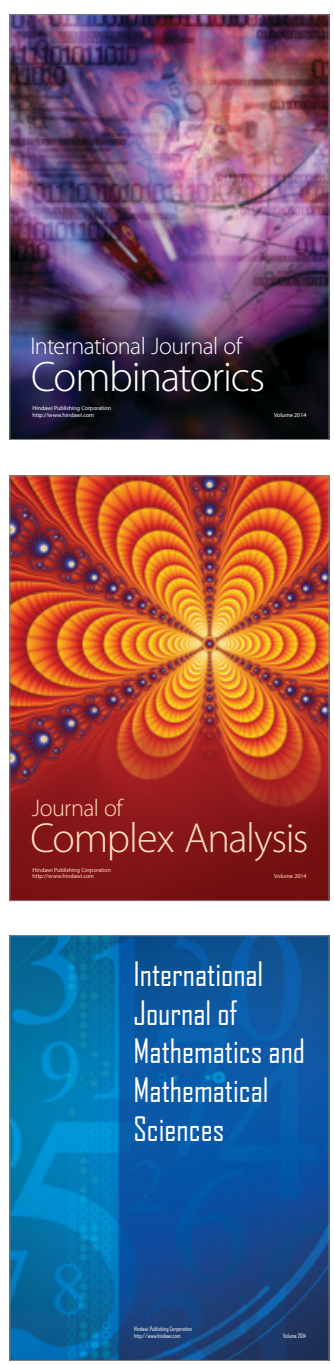
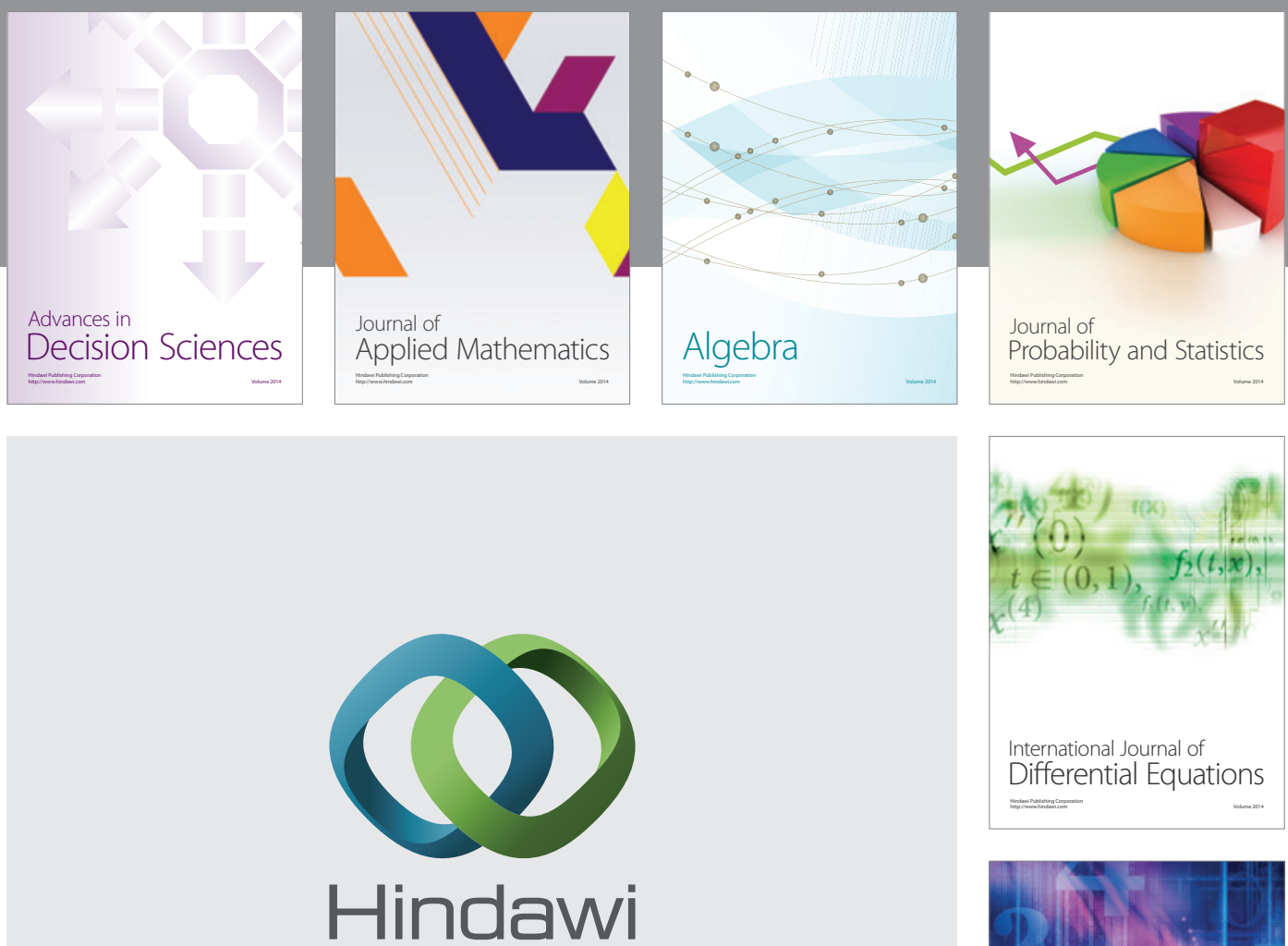

Submit your manuscripts at http://www.hindawi.com
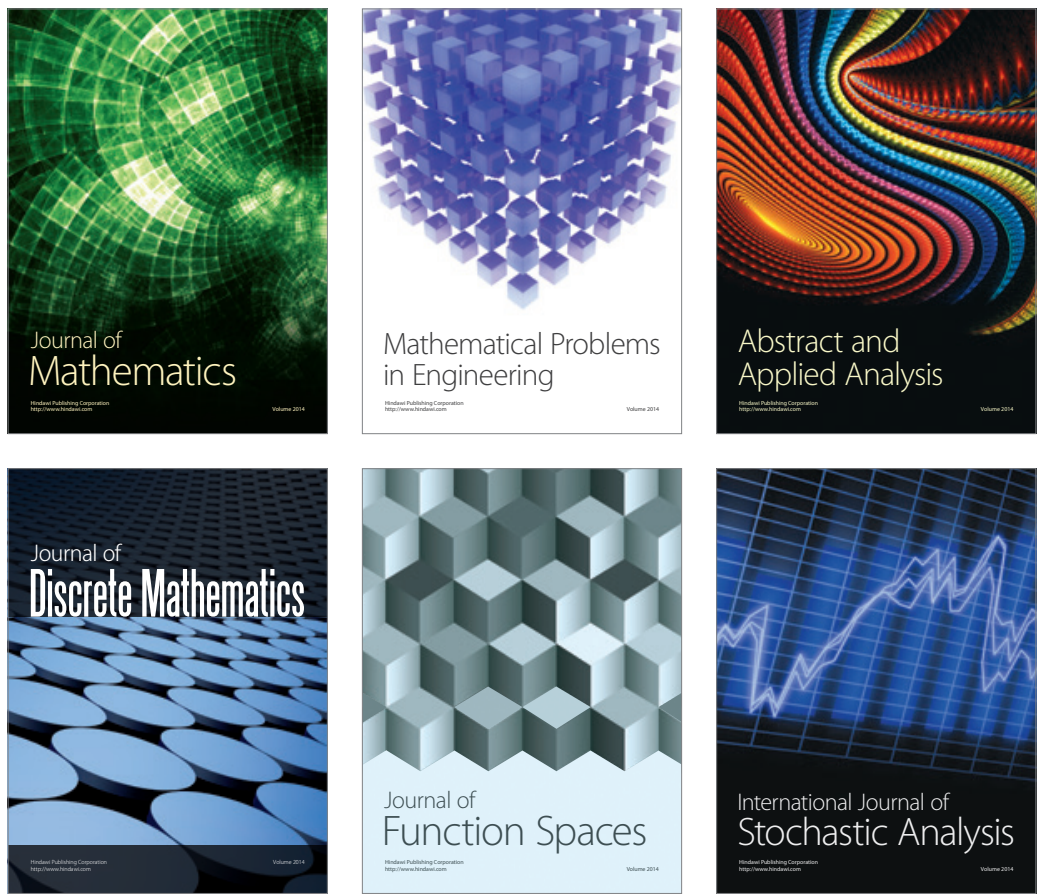

Journal of

Function Spaces

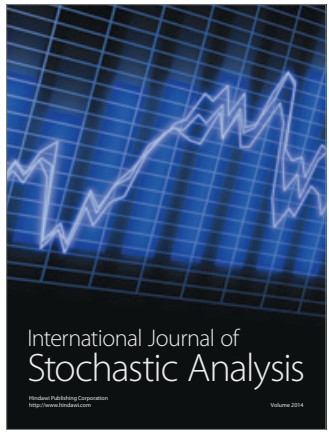

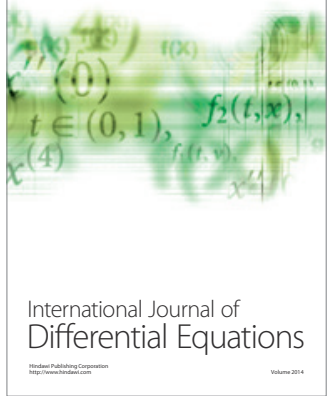
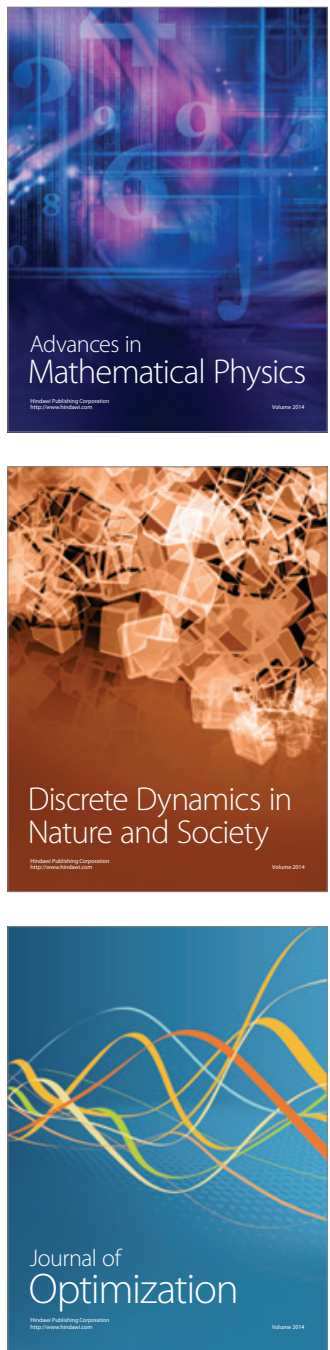